Article

\title{
On Wong Type Contractions
}

\author{
Erdal Karapınar $1,2, *\left(\mathbb{D}\right.$ and Andreea Fulga ${ }^{3, *}$
}

1 Department of Medical Research, China Medical University Hospital, China Medical University, Taichung 40402, Taiwan

2 Department of Mathematics, Çankaya University, Ankara 06790, Turkey

3 Department of Mathematics and Computer Science, Transilvania University of Brasov, 500036 Brasov, Romania

* Correspondence: karapinar@mail.cmuh.org.tw or erdalkarapinar@yahoo.com (E.K.); afulga@unitbv.ro (A.F.)

Received: 18 March 2020; Accepted: 21 April 2020; Published: 23 April 2020

Abstract: In this paper, by using admissible mapping, Wong type contraction mappings are extended and investigated in the framework of quasi-metric spaces to guarantee the existence of fixed points. We consider examples to illustrate the main results. We also demonstrate that the main results of the paper cover several existing results in the literature.

Keywords: Wong type contraction; quasi-metric spaces; fixed point

\section{Introduction and Preliminaries}

In 1974, Wong [1] announced an interesting extension of renowned Banach's contraction principle via auxiliary functions $\alpha_{i}:(0, \infty) \rightarrow[0, \infty)$. In this short note we aim to transform the result of Wong [1] in a weaker abstract space, namely quasi-metric space.

Below, we recall the fundamental notions that were used by Wong [1] to express his main result.

Definition 1. Suppose that there exist functions $f_{i}:(0, \infty) \rightarrow[0, \infty), i=1,2,3,4,5$, such that

(i) each $f_{i}$ is upper semi-continuous from the right;

(ii) $\sum_{i=1}^{5} f_{i}(u)<u$, for any $u>0$.

Then, $\left\{f_{i}\right\}_{i=1}^{5}$ is said to be a set of Wong (auxiliary) functions.

We shall state a Wong type contraction in which Wong auxiliary functions play a key role.

Definition 2. Let $A$ be a self-mapping on a metric space $(\mathcal{M}, d)$ and $\left\{f_{i}\right\}_{i=1}^{5}$ be a set of Wong (auxiliary) functions. We say that $A$ is a Wong type contraction if the following inequality holds:

$$
d(A p, A q) \leq a_{1} d(p, q)+a_{2} d(p, A p)+a_{3} d(q, A q)+a_{4} d(p, A q)+a_{5} d(A p, q)
$$

for any $p, q \in \mathcal{M}$ with $p \neq q$ where $a_{i}=f_{i}(d(p, q)) / d(p, q)$.

The following is the outstanding result in [1].

Theorem 1. [1] If a self-mapping $A$, on a complete metric space $(\mathcal{M}, d)$, is a Wong type contraction, then $A$ has exactly one fixed point. 
For the sake of self-containment of this note, we shall recollect some basic concepts of quasi-metric space. For more details, we refer the reader to [2-4].

Definition 3. Let $\mathcal{M}$ be a set and $\omega: \mathcal{M} \times \mathcal{M} \rightarrow[0, \infty)$ a function such that:

$\left(\omega_{1}\right) \omega(p, q)=0 \Leftrightarrow p=q$

$\left(\omega_{2}\right) \omega(p, s) \leq \omega(p, q)+\omega(q, s)$, for all $p, q, s \in \mathcal{M}$.

The function $\omega$ is called a quasi-metric and the pair $(\mathcal{M}, \omega)$ is a quasi-metric space.

If $\omega(p, q)=\omega(q, p)$, then $(\mathcal{M}, \omega)$ becomes a metric space and so, any metric space is a quasi-metric space, but the converse is not generally true.

Definition 4. Let $(\mathcal{M}, \omega)$ be a quasi-metric space and $\left\{p_{n}\right\}$ be a sequence in $\mathcal{M}$. We say that the sequence $\left\{p_{n}\right\}$ converges to $p \in \mathcal{M}\left(p_{n} \rightarrow p\right)$ if and only if

$$
\lim _{n \rightarrow \infty} \omega\left(p_{n}, p\right)=\lim _{n \rightarrow \infty} \omega\left(p, p_{n}\right)=0 .
$$

Remark 1. In a quasi-metric space $(\mathcal{M}, \omega)$, the limit for a convergent sequence is unique. If the sequence $\left\{p_{n}\right\}$ converges to $p \in \mathcal{M}$, we have for all $q \in \mathcal{M}$

$$
\lim _{n \rightarrow \infty} \omega\left(p_{n}, q\right)=\omega(p, q) \text { and } \lim _{n \rightarrow \infty} \omega\left(q, p_{n}\right)=\omega(q, p) .
$$

Definition 5. Let $(\mathcal{M}, \omega)$ be a quasi-metric space and $\left\{p_{n}\right\}$ be a sequence in $\mathcal{M}$. We say that the sequence $\left\{p_{n}\right\}$ is:

$\left(R_{C}\right)$ left-Cauchy if and only if for every $\varepsilon>0$ there exists a positive integer $N=N(\varepsilon)$ such that $\omega\left(p_{n}, p_{m}\right)<\varepsilon$ for all $n \geq m>N$.

$\left(L_{C}\right)$ right-Cauchy if and only if for every $\varepsilon>0$ there exists a positive integer $N=N(\varepsilon)$ such that $\omega\left(p_{n}, p_{m}\right)<\varepsilon$ for all $m \geq n>N$.

(C) Cauchy if and only if for every $\varepsilon>0$ there exists a positive integer $N=N(\varepsilon)$ such that $\omega\left(p_{n}, p_{m}\right)<\varepsilon$ for all $m, n>N$.

Remark 2. In a quasi-metric space a sequence $\left\{p_{n}\right\}$ is Cauchy if and only if it is left-Cauchy and right-Cauchy.

Definition 6. The quasi-metric space $(\mathcal{M}, \omega)$ is said to be:

$\left(c_{L}\right)$ left-complete if and only if each left-Cauchy sequence in $\mathcal{M}$ is convergent.

$\left(c_{R}\right)$ right-complete if and only if each right-Cauchy sequence in $\mathcal{M}$ is convergent.

(c) complete if and only if each Cauchy sequence in $\mathcal{M}$ is convergent.

Definition 7. In a quasi-metric space $(\mathcal{M}, \omega)$ a map $A: \mathcal{M} \rightarrow \mathcal{M}$ is continuous if, for each sequence $\left\{p_{n}\right\}$ in $\mathcal{M}$ converging to $p \in \mathcal{M}$, the sequence $\left\{A p_{n}\right\}$ converges to $A p$, that is,

$$
\lim _{n \rightarrow \infty} \omega\left(A p_{n}, A p\right)=\lim _{n \rightarrow \infty} \omega\left(A p, A p_{n}\right)=0
$$

Inspired by the interesting notion of $\alpha$-admissible mappings [5,6], we shall introduce $\alpha_{r}$-admissible. Let $\alpha: \mathcal{M} \times \mathcal{M} \rightarrow[0, \infty)$ and $r \geq 1$. We say that a map $A: \mathcal{M} \rightarrow \mathcal{M}$ is $\alpha_{r}$-admissible if for any $p, q \in \mathcal{M}$

$$
\alpha(p, q) \geq r \text { implies } \alpha(A p, A q) \geq r .
$$




\section{Main Results}

In this section, first we recall the notion of $\Delta$-symmetric [7], see also [8].

Definition 8. Let $\Delta$ be a positive real number, that is, $\Delta>0$. A quasi-metric space $(X, q)$ is called $\Delta$-symmetric if

$$
q(y, x) \leq \Delta q(x, y) \quad \text { for all } x, y \in X
$$

If $X$ is not reduced to a single point, we can find $x, y \in X$ such that $q(x, y)>0$. Therefore, $0<q(x, y) \leq \Delta q(y, x) \leq \Delta^{2} q(x, y)$. Hence, $\Delta \geq 1$.

Remark 3. Note that for $\Delta=1$, every metric space forms a symmetric space. In other words, a quasi-metric space is a metric space if, and only if, it is 1-symmetric.

Example 1. Let $X=\mathbb{R}$ and let define

$$
q(x, y)= \begin{cases}2(x-y), & \text { if } x \geq y \\ y-x, & \text { if } x<y .\end{cases}
$$

Then $(X, q)$ is a complete 2 -symmetric quasi-metric space, but it is not a metric space.

Proposition 1. Let $(\mathcal{M}, \omega)$ be a quasi-metric space and $A: \mathcal{M} \rightarrow \mathcal{M}$ be an $\alpha_{r}$-admissible function. Let $p_{0}$ be fixed in $\mathcal{M}$ and the sequence $\left\{p_{n}\right\}$ be defined as $p_{n}=A p_{n-1}$. If $\alpha\left(p_{0}, p_{1}\right) \geq r$ and $\alpha\left(p_{1}, p_{0}\right) \geq r$ then $\alpha\left(p_{n}, p_{n+1}\right) \geq r$, and $\alpha\left(p_{n+1}, p_{n}\right) \geq r$.

Proof. Since $A$ is $\alpha_{r}$-admissible, $\alpha\left(p_{0}, p_{1}\right) \geq r$ implies $\alpha\left(p_{1}, p_{2}\right)=\alpha\left(A p_{0}, A p_{1}\right) \geq r$. Repeating this procedure, we find that for all natural numbers $n$, indeed,

$$
\alpha\left(p_{n}, p_{n+1}\right) \geq r
$$

Similarly, we derive that $\alpha\left(p_{n+1}, p_{n}\right) \geq r$.

In connection with this notion, we consider the following definitions:

(R) Let $\left\{p_{n}\right\}$ be a sequence in $\mathcal{M}$ such that $p_{n} \rightarrow p$ as $n \rightarrow \infty, p \in \mathcal{M}$. If there exists a subsequence $\left\{p_{k(n)}\right\}$ of $\left\{p_{n}\right\}$ such that $\alpha\left(p_{n}, p_{n+1}\right) \geq r$ implies $\alpha\left(p_{k(n)}, p\right) \geq r$ for any $n \geq 1$, then we say that the space $\mathcal{M}$ is $\alpha_{r}$-regular.

(U) For $r \geq 1$, if $\alpha(p, q) \geq r$ for all $p, q \in$ Fix $(A)$, then we say that $\alpha$ satisfies the (U)-condition, where Fix $(A)$ denotes the set of fixed points of $A$.

Theorem 2. Let $(\mathcal{M}, \omega)$ be a complete $\Delta$-symmetric quasi-metric space, a function $A: \mathcal{M} \rightarrow \mathcal{M}$ and $r \geq 1$. Suppose that there exist the functions $\beta_{i}, \gamma_{i}:(0, \infty) \rightarrow[0, \infty), \beta_{i}(u)=\frac{\gamma_{i}(u)}{u}, i=1,2,3,4$ such that:

(i) the map $A$ is $\alpha_{r}$-admissible and there exists $p_{0} \in \mathcal{M}$ such that $\alpha\left(p_{0}, A p_{0}\right) \geq r$ and $\alpha\left(A p_{0}, p_{0}\right) \geq r$;

(ii) $\mathcal{M}$ is $\alpha_{r}$-regular;

(iii) each $\gamma_{i}$ is upper semi-continuous from the right;

(iv) $\sum_{i=1}^{4} \beta_{i}(u)<r$ for any $u>0$; 
(v) for any $p, q \in \mathcal{M}$ with $p \neq q$

$$
\begin{gathered}
\alpha(p, q) \omega(A p, A q) \leq \beta_{1}(\omega(p, q)) \omega(p, q)+\beta_{2}(\omega(p, q)) \omega(p, A p)+\beta_{3}(\omega(p, q)) \omega(q, A q) \\
+\beta_{4}(\omega(p, q)) \frac{\omega(p, A q)+\omega(A p, q)}{2} .
\end{gathered}
$$

Then A has a fixed point.

Proof. Starting with an arbitrary point $p_{0}$ from $\mathcal{M}$ we construct the sequence $\left\{p_{n}\right\}$ as follows:

$$
p_{1}=A p_{0} \text { and } p_{n+1}=A^{n} p_{0} \text { for all } n \in \mathbb{N} .
$$

It is worth noting that it is interesting to assume that any adjacent terms in the sequence $\left\{p_{n}\right\}$ are different. Indeed, on the contrary, if there is $n_{0}$ such that $p_{n_{0}}=p_{n_{0}+1}$ then from the definition of sequence $\left\{p_{n}\right\}$ we get $p_{n_{0}}=p_{n_{0}+1}=A p_{n_{0}}$. Consequently, $p_{n_{0}}$ is a fixed point of $A$ and there is nothing to prove it. Accordingly, from now on, we consider $\omega\left(p_{n}, p_{n+1}\right)>0$, for any $n \in \mathbb{N}$.

By the condition (5), replacing $p$ by $p_{n}$ and $q$ by $p_{n+1}$ and taking into account Proposition 1 we get

$$
\begin{aligned}
0< & r \omega\left(p_{n+1}, p_{n+2}\right)=r \omega\left(A p_{n}, A p_{n+1}\right) \leq \alpha\left(p_{n}, p_{n+1}\right) \omega\left(A p_{n}, A p_{n+1}\right) \\
\leq & \beta_{1}\left(\omega\left(p_{n}, p_{n+1}\right)\right) \omega\left(p_{n}, p_{n+1}\right)+\beta_{2}\left(\omega\left(p_{n}, p_{n+1}\right)\right) \omega\left(p_{n}, A p_{n}\right) \\
& +\beta_{3}\left(\omega\left(p_{n}, p_{n+1}\right)\right) \omega\left(p_{n+1}, A p_{n+1}\right)+\beta_{4}\left(\omega\left(p_{n}, p_{n+1}\right)\right) \frac{\omega\left(p_{n}, A p_{n+1}\right)+\omega\left(A p_{n}, p_{n+1}\right)}{2} \\
\leq & \beta_{1}\left(\omega\left(p_{n}, p_{n+1}\right)\right) \omega\left(p_{n}, p_{n+1}\right)+\beta_{2}\left(\omega\left(p_{n}, p_{n+1}\right)\right) \omega\left(p_{n}, p_{n+1}\right) \\
& +\beta_{3}\left(\omega\left(p_{n}, p_{n+1}\right)\right) \omega\left(p_{n+1}, p_{n+2}\right)+\beta_{4}\left(\omega\left(p_{n}, p_{n+1}\right)\right) \frac{\omega\left(p_{n}, p_{n+2}\right)+\omega\left(p_{n+1}, p_{n+1}\right)}{2}
\end{aligned}
$$

Since $\beta_{i}(u)=\frac{\gamma_{i}(u)}{u}$ for $i=1,2,3,4$, and by using the triangle inequality, the previous inequality yields that

$$
\begin{aligned}
r \omega\left(p_{n+1}, p_{n+2}\right) \omega\left(p_{n}, p_{n+1}\right) \leq & \gamma_{1}\left(\omega\left(p_{n}, p_{n+1}\right)\right) \omega\left(p_{n}, p_{n+1}\right)+\gamma_{2}\left(\omega\left(p_{n}, p_{n+1}\right)\right) \omega\left(p_{n}, p_{n+1}\right) \\
& +\gamma_{3}\left(\omega\left(p_{n}, p_{n+1}\right)\right) \omega\left(p_{n+1}, p_{n+2}\right) \\
& +\gamma_{4}\left(\omega\left(p_{n}, p_{n+1}\right)\right) \frac{\omega\left(p_{n}, p_{n+1}\right)+\omega\left(p_{n+1}, p_{n+2}\right)}{2} .
\end{aligned}
$$

For simplicity, let $a_{n}=\omega\left(p_{n}, p_{n+1}\right)$. Accordingly, the inequality above turns into

$$
r \cdot a_{n+1} a_{n} \leq \gamma_{1}\left(a_{n}\right) a_{n}+\gamma_{2}\left(a_{n}\right) a_{n}+\gamma_{3}\left(a_{n}\right) a_{n+1}+\gamma_{4}\left(a_{n}\right) \frac{a_{n}+a_{n+1}}{2} .
$$

By elementary computation, we derive from the inequality above that

$$
a_{n+1} \leq \frac{\gamma_{1}\left(a_{n}\right)+\gamma_{2}\left(a_{n}\right)+\gamma_{4}\left(a_{n}\right) / 2}{r \cdot a_{n}-\gamma_{3}\left(a_{n}\right)-\gamma_{4}\left(a_{n}\right) / 2} a_{n}=\gamma\left(a_{n}\right),
$$

where for $u>0$,

$$
\gamma(u)=\frac{\gamma_{1}(u)+\gamma_{2}(u)+\gamma_{4}(u) / 2}{r \cdot u-\gamma_{3}(u)-\gamma_{4}(u) / 2} u
$$

Since from (iv), $\gamma(u)<u$ for all $u>0$ we obtain $a_{n+1}<a_{n}$ and so, the sequence $\left\{a_{n}\right\}$ is decreasing. Thus, there exists a point $a \in[0, \infty)$ such that $\lim _{n \rightarrow \infty} a_{n}=a$. On the other hand, since each function $\gamma_{i}$ is upper semi-continuous from the right, the mapping $\gamma$ is also upper semi-continuous from the right, 
so $\lim _{n \rightarrow \infty} \gamma\left(a_{n}\right)=a$. We will prove that $a=0$. Indeed, if we suppose that $a>0$, then, from the expression (6), we have

$$
a=\lim _{n \rightarrow \infty} a_{n+1} \leq \limsup _{n \rightarrow \infty} \gamma\left(a_{n}\right) \leq \gamma(a)<a,
$$

which is a contradiction. We conclude that the sequence $\left\{a_{n}\right\}$ converges to 0 .

Let us consider now, in relation (5), $p=p_{n+1}$ and $q=p_{n}$. We find

$$
\begin{aligned}
0< & r \omega\left(p_{n+2}, p_{n+1}\right)=r \omega\left(A p_{n+1}, A p_{n}\right) \leq \alpha\left(p_{n+1}, p_{n}\right) \omega\left(A p_{n+1}, A p_{n}\right) \\
\leq & \beta_{1}\left(\omega\left(p_{n+1}, p_{n}\right)\right) \omega\left(p_{n+1}, p_{n}\right)+\beta_{2}\left(\omega\left(p_{n+1}, A p_{n+1}\right)\right) \omega\left(p_{n+1}, A p_{n+1}\right) \\
& +\beta_{3}\left(\omega\left(p_{n+1}, p_{n}\right)\right) \omega\left(p_{n}, A p_{n}\right)+\beta_{4}\left(\omega\left(p_{n+1}, p_{n}\right)\right) \frac{\omega\left(p_{n+1}, A p_{n}\right)+\omega\left(A p_{n+1}, p_{n}\right)}{2} \\
\leq & \beta_{1}\left(\omega\left(p_{n+1}, p_{n}\right)\right) \omega\left(p_{n+1}, p_{n}\right)+\beta_{2}\left(\omega\left(p_{n+1}, p_{n}\right)\right) \omega\left(p_{n+1}, p_{n+2}\right) \\
& +\beta_{3}\left(\omega\left(p_{n+1}, p_{n}\right)\right) \omega\left(p_{n}, p_{n+1}\right)+\beta_{4}\left(\omega\left(p_{n+1}, p_{n}\right)\right) \frac{\omega\left(p_{n+1}, p_{n+1}\right)+\omega\left(p_{n+2}, p_{n}\right)}{2}
\end{aligned}
$$

or,

$$
\begin{aligned}
r \omega\left(p_{n+2}, p_{n+1}\right) \omega\left(p_{n+1}, p_{n}\right) & \leq \gamma_{1}\left(\omega\left(p_{n+1}, p_{n}\right)\right) \omega\left(p_{n+1}, p_{n}\right)+\gamma_{2}\left(\omega\left(p_{n+1}, p_{n+2}\right)\right) \omega\left(p_{n+1}, p_{n}\right) \\
& +\gamma_{3}\left(\omega\left(p_{n+1}, p_{n}\right)\right) \omega\left(p_{n}, p_{n+1}\right) \\
& +\gamma_{4}\left(\omega\left(p_{n+1}, p_{n}\right)\right) \frac{\omega\left(p_{n+2}, p_{n+1}\right)+\omega\left(p_{n+1}, p_{n+2}\right)}{2} .
\end{aligned}
$$

On the other hand, denoting by $b_{n}=\omega\left(p_{n+1}, p_{n}\right)$ and keeping in my that it is $\Delta$-symmetric, there exists $\Delta>0$ such that

$$
\frac{1}{\Delta} a_{n} \leq b_{n} \leq \Delta a_{n}
$$

Letting $n \rightarrow \infty$, by using the "Squeeze Theorem" and taking into account that the sequence $\left\{a_{n}\right\}$ converges to 0 we get $\lim _{n \rightarrow \infty} b_{n}=\lim _{n \rightarrow \infty} \omega\left(p_{n+1}, p_{n}\right)=0$.

As a next step, we shall show that the sequence $\left\{p_{n}\right\}$ is Cauchy, that is left-Cauchy and right-Cauchy sequence in the quasi-metric space $(\mathcal{M}, \omega)$.

Suppose it would not be so, which means that there exists $\varepsilon>0$ and two sequences $k(n)$ and $l(n)$ such that for any $n \in \mathbb{N}$

$$
k(n)>l(n)>n, \quad \omega\left(p_{k(n)}, p_{l(n)}\right) \geq \varepsilon, \text { and } \omega\left(p_{k(n)-1}, p_{l(n)}\right)<\varepsilon .
$$

Then, due to the triangle inequality, we get

$$
\varepsilon \leq \omega\left(p_{k(n)}, p_{l(n)}\right) \leq \omega\left(p_{k(n)}, p_{k(n)-1}\right)+\omega\left(p_{k(n)-1}, p_{l(n)}\right) \leq \omega\left(p_{k(n)}, p_{k(n)-1}\right)+\varepsilon .
$$

or, if we denote by $c_{n}=\omega\left(p_{k(n)}, p_{l(n)}\right)$

$$
\varepsilon \leq c_{n} \leq b_{n}+\varepsilon
$$

Since $b_{n} \rightarrow 0$, taking $n \rightarrow \infty$ in (7) we obtain

$$
\varepsilon \leq \lim _{n \rightarrow \infty} c_{n} \leq \varepsilon
$$

so, $\lim _{n \rightarrow \infty} c_{n}=\lim _{n \rightarrow \infty} \omega\left(p_{k(n)}, p_{l(n)}\right)=\varepsilon$. Again, from the triangle inequality, 


$$
\begin{aligned}
\omega\left(p_{k(n)+1}, p_{l(n)+1}\right) & \leq \omega\left(p_{k(n)+1}, p_{k(n)}\right)+\omega\left(p_{k(n)}, p_{l(n)}\right)+\omega\left(p_{l(n)}, p_{l(n)+1}\right) \\
& \leq b_{k(n)}+\omega\left(p_{k(n)}, p_{l(n)}\right)+a_{l(n)}
\end{aligned}
$$

and also

$$
\begin{aligned}
\omega\left(p_{k(n)}, p_{l(n)}\right) & \leq \omega\left(p_{k(n)}, p_{k(n)+1}\right)+\omega\left(p_{k(n)+1}, p_{l(n)+1}\right)+\omega\left(p_{l(n)+1}, p_{l(n)}\right) \\
& \leq a_{k(n)}+\omega\left(p_{k(n)+1}, p_{l(n)+1}\right)+b_{l(n)} .
\end{aligned}
$$

Combining (8) and (9)

$$
\omega\left(p_{k(n)}, p_{l(n)}\right)-a_{k(n)}-b_{l(n)} \leq \omega\left(p_{k(n)+1}, p_{l(n)+1}\right) \leq b_{k(n)}+\omega\left(p_{k(n)}, p_{l(n)}\right)+a_{l(n)} .
$$

Letting $n \rightarrow \infty$ we get $\lim _{n \rightarrow \infty} \omega\left(p_{k(n)+1}, p_{l(n)+1}\right)=\varepsilon$. On the other hand, by $(\mathrm{v})$,

$$
\begin{aligned}
r \omega\left(A p_{k(n)}, A p_{l(n)}\right) \leq & \alpha\left(p_{k(n)}, p_{l(n)}\right) \omega\left(A p_{k(n)}, A p_{l(n)}\right) \leq \beta_{1}\left(\omega\left(p_{k(n)}, p_{l(n)}\right)\right) \omega\left(p_{k(n)}, p_{l(n)}\right) \\
& +\beta_{2}\left(\omega\left(p_{k(n)}, p_{l(n)}\right)\right) \omega\left(p_{k(n)}, A p_{k(n)}\right)+\beta_{3}\left(\omega\left(p_{k(n)}, p_{l(n)}\right)\right) \omega\left(p_{(n)}, A p_{l(n)}\right) \\
& +\beta_{4}\left(\omega\left(A p_{k(n)}, A p_{l(n)}\right)\right) \frac{\omega\left(p_{k(n)}, A p_{l(n)}\right)+\omega\left(A p_{k(n)}, p_{l(n)}\right)}{2} .
\end{aligned}
$$

Taking into account the definition of function $\beta$, and using again the triangle inequality,

$$
r c_{n+1} c_{n} \leq \gamma_{1}\left(c_{n}\right) c_{n}+\gamma_{2}\left(c_{n}\right) a_{k(n)}+\gamma_{3}\left(c_{n}\right) a_{l(n)}+\gamma_{4}\left(c_{n}\right) \frac{2 c_{n}+a_{l(n)}+b_{k(n)}}{2}
$$

By letting $n \rightarrow \infty$, since the functions $\gamma_{i}$ are upper semi-continuous and using (iv)

$$
r \cdot \varepsilon^{2} \leq\left[\gamma_{1}(\varepsilon)+\gamma_{4}(\varepsilon)\right] \varepsilon<r \cdot \varepsilon^{2}
$$

which is a contradiction. Thus $\left\{p_{n}\right\}$ is a left-Cauchy sequence. Analogously, it can be shown that $\left\{p_{n}\right\}$ is right-Cauchy and we can conclude that $\left\{p_{n}\right\}$ is a Cauchy sequence in the complete quasi-metric space $(\mathcal{M}, \omega)$. This implies that the sequence $\left\{p_{n}\right\}$ converges to some point $p^{*}$, that is

$$
\lim _{n \rightarrow \infty} \omega\left(p_{n}, p^{*}\right)=\lim _{n \rightarrow \infty} \omega\left(p^{*}, p_{n}\right)=0 .
$$

We shall prove now that $p^{*}$, is a fixed point of $A$. Since the sequence $\left\{a_{n}\right\}$ is strictly decreasing and $a_{n}>0$ for any natural number $n$, we can find a subsequence $p_{k(n)}$ such that $\omega\left(p_{k(n)}, p^{*}\right)>0$. Also from (ii), the space $\left(\mathcal{M}, p^{*}\right)$ is $\alpha$-regular, that is $\alpha\left(p_{k(n)}, p^{*}\right) \geq r$ for any $r \geq 1$. Then, we have

$$
\begin{aligned}
r \omega\left(p_{k(n+1)}, A p^{*}\right)= & r \cdot \omega\left(A p_{k(n)}, A p^{*}\right) \leq r \cdot \alpha\left(p_{k(n)}, p^{*}\right) \omega\left(A p_{k(n)}, A p^{*}\right) \\
\leq & \beta_{1}\left(\omega\left(p_{k(n)}\right), p^{*}\right) \omega\left(p_{k(n)}, p^{*}\right)+\beta_{2}\left(\omega\left(p_{k(n)}, p^{*}\right)\right) \omega\left(p_{k(n)}, A p_{k(n)}\right) \\
& +\beta_{3}\left(\omega\left(p_{k(n)}, p^{*}\right)\right) \omega\left(p_{k(n)}, A p_{k(n)}\right)+\beta_{4}\left(\omega\left(p_{k(n)}, p^{*}\right)\right) \frac{\left.\omega\left(p_{k(n)}, A p^{*}\right)+\omega\left(A p_{k(n)}, p^{*}\right)\right)}{2} \\
\leq & \beta_{1}\left(\omega\left(p_{k(n)}, p^{*}\right)\right) \omega\left(p_{k(n)}, p^{*}\right)+\beta_{2}\left(\omega\left(p_{k(n)}, p^{*}\right)\right) \omega\left(p_{k(n)}, p_{k(n)+1}\right) \\
& +\beta_{3}\left(\omega\left(p_{k(n)}\right), p^{*}\right) \omega\left(p^{*}, A p^{*}\right)+\beta_{4}\left(\omega\left(p_{k(n)}, p^{*}\right)\right) \frac{\omega\left(p_{k(n)}, A p^{*}\right)+\omega\left(p_{k(n)+1}, p^{*}\right)}{2} \\
\leq & \beta_{1}\left(\omega\left(p_{k(n)}, p^{*}\right)\right) \omega\left(p_{k(n)}, p^{*}\right)+\beta_{2}\left(\omega\left(p_{k(n)}, p^{*}\right)\right) \omega\left(p_{k(n)}, p_{k(n)+1}\right) \\
& +\beta_{3}\left(\omega\left(p_{k(n)}, p^{*}\right) \omega\left(p^{*}, A p^{*}\right)+\beta_{4}\left(\omega\left(p_{k(n)}, p^{*}\right)\right) \frac{\omega\left(p_{k(n)}, p^{*}\right)+\omega\left(\omega, A p^{*}\right)+\omega\left(p_{k(n)+1}, p^{*}\right)}{2}\right.
\end{aligned}
$$


By denoting $\omega_{n}=\omega\left(p_{k(n)}, p^{*}\right)$, we can rewrite as

$$
r \cdot \omega\left(p_{k(n)+1}, A p^{*}\right) \leq \beta_{1}\left(\omega_{n}\right) \omega_{n}+\beta_{2}\left(\omega_{n}\right) a_{k(n)}+\beta_{3}\left(\omega_{n}\right) \omega\left(p^{*}, A p^{*}\right)+\beta_{4}\left(\omega_{n}\right) \frac{\omega\left(p^{*}, A p^{*}\right)+2 \omega_{n}+b_{k(n)}}{2},
$$

or

$$
\begin{aligned}
r \cdot \omega\left(p_{k(n)+1}, A p^{*}\right) & \leq \frac{\gamma_{3}\left(\omega_{n}\right)+\frac{\gamma_{4}\left(\omega_{n}\right)}{2}}{\omega_{n}} \omega\left(p^{*}, A p^{*}\right)+\frac{1}{\omega_{n}}\left[\gamma_{1}\left(\omega_{n}\right) \omega_{n}+\gamma_{4}\left(\omega_{n}\right)\left(\omega_{n}+\frac{b_{k(n)}}{2}\right)+\gamma_{2}\left(\omega_{n}\right) a_{k(n)}\right] \\
& <r \cdot \omega\left(p^{*}, A p^{*}\right)+O(n),
\end{aligned}
$$

where

$$
O(n)=\frac{1}{\omega_{n}}\left[\gamma_{1}\left(\omega_{n}\right) \omega_{n}+\gamma_{4}\left(\omega_{n}\right)\left(\omega_{n}+\frac{b_{k(n)}}{2}\right)+\gamma_{2}\left(\omega_{n}\right) a_{k(n)}\right] .
$$

Taking $n \rightarrow \infty$ in the previous inequality, since $\lim _{n \rightarrow \infty} O(n)=0$ we obtain that

$$
r \cdot \omega\left(p^{*}, A p^{*}\right)<r \cdot \omega\left(p^{*}, A p^{*}\right),
$$

a contradiction. We conclude that $A p^{*}=p^{*}$, that is $p^{*}$ is a fixed point of function $A$.

Example 2. Let $\mathcal{M}=[0,2] \cup\{3\}$ and $\omega: \mathcal{M} \times \mathcal{M} \rightarrow[0, \infty)$ be defined by

$$
\omega(p, q)= \begin{cases}0 & \text { for } p=q \\ 3 & \text { for } p \in[0,2], q=3 \\ 1 & \text { otherwise }\end{cases}
$$

Define the function $A: \mathcal{M} \rightarrow \mathcal{M}$ by

$$
A p=\left\{\begin{aligned}
\frac{1}{2} & \text { for } p \in[0,1) \cup\{3\} \\
p+\ln p & \text { for } p \in[1,2]
\end{aligned}\right.
$$

and $\alpha: \mathcal{M} \times \mathcal{M} \rightarrow[0, \infty)$ by

$$
\alpha(p, q)= \begin{cases}4 & \text { for } p, q \in[0,1) \\ 3 & \text { for } p \in[0,1), q=3 \\ 0 & \text { otherwise }\end{cases}
$$

We take also $r=3$ and $\beta_{i}, \gamma_{i}:(0, \infty) \rightarrow[0, \infty), i=1,2,3,4$ defined by $\beta_{i}(u)=\frac{\gamma_{i}(u)}{u}$, where $\gamma_{1}(u)=\frac{u^{2}}{u+1}$, $\gamma_{4}(u)=\frac{1}{2} u, \gamma_{2}(u)=\gamma_{3}(u)=0$.

Obviously, $\sum \beta_{i}(u)=\frac{u}{u+1}+\frac{1}{2}<3$ for any $u>0$. On the other hand, since $A(p)<1$ for any $p \in[0,1)$ and $A(3)=1 / 2$ is easy to see that $A$ is $\alpha$-admissible and the space $\mathcal{M}$ is $\alpha$-regular. Moreover (vi) holds for $\Delta=3$. We are only interested in the following cases:

(i) For $p, q \in[0,1)$ we have $\omega(p, q)=1, \omega(p, A q)=1, \omega(q, A p)=1, \beta_{1}(\omega(p, q))=\frac{1}{2}, \beta_{4}(\omega(p, 0))=\frac{1}{2}$. Thus,

$$
\alpha(p, 0) \omega(A p, A q)=0<\frac{1}{2}+\frac{1}{2}=\beta_{1}(\omega(p, q)) \omega(p, q)+\beta_{4}(\omega(p, q))[\omega(p, A q)+(A p, q)] / 2 .
$$


(ii) For $p \in[0,1)$ and $q=3$, we have $\omega(p, 3)=3, \omega(p, A(3))=\omega(p, 1 / 4)=1, \omega(A p, 3)=3, \beta_{1}(\omega(p, 3))=\frac{3}{4}$, $\beta_{4}(\omega(p, 3))=\frac{1}{2}$. Thus,

$$
\alpha(p, 3) \omega(A p, A(3))=0<\frac{3}{4}+\frac{4}{2}=\beta_{1}(\omega(p, 3)) \omega(p, 3)+\beta_{4}(\omega(p, 3))[\omega(p, A(3))+\omega(A p, 3)] / 2 .
$$

Hence, in all cases, the conditions of the Theorem 2 are satisfied and the function $A$ has two fixed points $p=1$ and $p=1 / 2$.

To ensure the uniqueness of the fixed point, we need to add an additional hypothesis. Thus, we obtain the following theorem:

Theorem 3. Additionally to the hypothesis of Theorem 2, if we suppose that the mapping $\alpha$ satisfies the $(U)$ condition, then the function A has exactly one fixed point.

Proof. Suppose that $A$ has two distinct fixed points $v, \mu$. Due the supplementary condition, we know that $\alpha(\nu, \mu) \geq r$, so replacing in (5) we get

$$
\begin{aligned}
r \omega(v, \mu)= & \cdot \omega(A v, A \mu) \leq \alpha(\nu, \mu) \omega(A v, A \mu) \leq \beta_{1}(\omega(\nu, \mu)) \omega(v, \mu)+\beta_{2}(\omega(\nu, \mu)) \omega(v, A v) \\
& \quad+\beta_{3}(\omega(\nu, \mu)) \omega(\mu, A \mu)+\beta_{4}(\omega(\nu, \mu)) \frac{\omega(\nu, A \mu)+\omega(A v, \mu)}{2} \\
= & {\left[\gamma_{1}(\omega(v, \mu)) \omega(\nu, \mu)+\gamma_{4}(\omega(\nu, \mu)) \frac{\omega(v, \mu)+\omega(v, \mu)}{2}\right] \cdot \frac{1}{\omega(\nu, \mu)} } \\
= & \gamma_{1}(\omega(\nu, \mu))+\gamma_{4}(\omega(\nu, \mu))<r \omega(\nu, \mu) .
\end{aligned}
$$

That contradiction shows us that $v=\mu$, that is, $A$ has exactly one fixed point.

Example 3. Let $\mathcal{M}=[0,1]$ and $\omega: \mathcal{M} \times \mathcal{M} \rightarrow[0, \infty)$ be defined by

$$
\omega(p, q)=\left\{\begin{aligned}
p-q & \text { for } p \geq q \\
\frac{1}{2}(q-p) & \text { for } p<q .
\end{aligned}\right.
$$

Then $(\mathcal{M}, \omega)$ is a complete quasi-metric space and it is easy to see that the assumption (vi) is satisfied for any $\Delta \geq 2$. Define $A: \mathcal{M} \rightarrow \mathcal{M}$ by

$$
A p=\left\{\begin{aligned}
\frac{p^{2}+p}{8} & \text { for } p \in[0,1) \\
0 & \text { for } p=1
\end{aligned}\right.
$$

and $\alpha: \mathcal{M} \times \mathcal{M} \rightarrow[0, \infty)$ by

$$
\alpha(p, q)= \begin{cases}3 & \text { for } p \in[0,1), q=1 \\ 2 & \text { for } p \in[0,1), q=0 \\ 0 & \text { otherwise }\end{cases}
$$

We take also $r=2$ and $\beta_{i}, \gamma_{i}:(0, \infty) \rightarrow[0, \infty), i=1,2,3,4$ defined by $\beta_{i}(u)=\frac{\gamma_{i}(u)}{u}$, where $\gamma_{1}(u)=\frac{u}{u+1}$, $\gamma_{3}(u)=\frac{2}{3} u, \gamma_{2}(u)=\gamma_{4}(u)=0$.

Obviously, $\sum \beta_{i}(u)=\frac{1}{u+1}+\frac{2}{3}<2$ for any $u>0$. On the other hand, since $A p<1$ for any $p \in[0,1)$ and $A(0)=A(1)=0$ is easy to see that $A$ is $\alpha$-admissible and the space $\mathcal{M}$ is $\alpha$-regular. We will consider the following cases: 
(i) For $p \in(0,1)$ and $q=0$, we have $\omega(p, 0)=p, \omega(0, A 0)=0, \beta_{1}(\omega(p, 0))=\frac{1}{1+p}, \beta_{3}(\omega(p, 0))=\frac{2}{3}$. Thus,

$$
\alpha(p, 0) \omega(A p, A(0))=2 \cdot \frac{p^{2}+p}{8}=\frac{p^{2}+p}{4} \leq \frac{p}{1+p}=\beta_{1}(\omega(p, 0)) \omega(p, 0)+\beta_{3}(\omega(p, 0)) \omega(0, A 0)
$$

(ii) For $p \in(0,1)$ and $q=1$, we have $\omega(p, 1)=\frac{1}{2}(1-p), \omega(1, A(1))=1, \beta_{1}(\omega(p, 1))=\frac{2}{3-p}, \beta_{3}(\omega(p, 1))=\frac{2}{3}$. Thus,

$$
\alpha(p, 1) \omega(A p, A(1))=3 \cdot \frac{p^{2}+p}{8} \leq \frac{1-p}{3-p}+\frac{2}{3}=\beta_{1}(\omega(p, 1)) \omega(p, 1)+\beta_{3}(\omega(p, 0)) \omega(1, A(1)) .
$$

For $p=q=1$ and $p=q=0$ the relation (5) is obviously satisfied, and the other cases are not interesting due to the choice of function $\alpha$. Therefore, the function $A$ has exactly one fixed point, namely $p=0$.

Letting $\alpha(p, q)=1$ and $r=1$ in Theorem 3 we obtain the following:

Corollary 1. Let $(\mathcal{M}, \omega)$ be a complete $\Delta$-symmetric quasi-metric space and a function $A: \mathcal{M} \rightarrow \mathcal{M}$. Suppose that there exist the functions $\beta_{i}, \gamma_{i}:(0, \infty) \rightarrow[0, \infty), \beta_{i}(u)=\frac{\gamma_{i}(u)}{u}, i=1,2,3,4$ such that:

(i) each $\gamma_{i}$ is upper semi-continuous from the right;

(ii) $\sum_{i=1}^{4} \beta_{i}(u)<1$ for any $u>0$;

(iii) for any $p, q \in \mathcal{M}$

$$
\begin{aligned}
\omega(A p, A q) \leq & \beta_{1}(\omega(p, q)) \omega(p, q)+\beta_{2}(\omega(p, q)) \omega(p, A p)+\beta_{3}(\omega(p, q)) \omega(q, A q) \\
& +\beta_{4}(\omega(p, q)) \frac{\omega(p, A q)+\omega(A p, q)}{2} .
\end{aligned}
$$

Then A has exactly one fixed point.

Letting $\beta_{2}=\beta_{3}=\beta_{4}=0$ we derive the following theorem:

Theorem 4. Let $(\mathcal{M}, \omega)$ be a complete quasi-metric space, a function $A: \mathcal{M} \rightarrow \mathcal{M}$ and $r \geq 1$. Suppose that there exist the functions $\beta_{1}, \gamma_{1}:(0, \infty) \rightarrow[0, \infty), \beta_{1}(u)=\frac{\gamma_{1}(u)}{u}$, such that:

(i) the map $A$ is $\alpha_{r}$-admissible and there exists $p_{0} \in \mathcal{M}$ such that $\alpha\left(p_{0}, A p_{0}\right) \geq r$ and $\alpha\left(A p_{0}, p_{0}\right) \geq r$;

(ii) $\mathcal{M}$ is $\alpha_{r}$-regular;

(iii) each $\gamma_{1}$ is upper semi-continuous from the right;

(iv) $\beta_{1}(u)<1$ for any $u>0$;

(v) for any $p, q \in \mathcal{M}$

$$
\alpha(p, q) \omega(A p, A q) \leq \beta_{1}(\omega(p, q)) \omega(p, q)
$$

Then A has a fixed point.

Theorem 5. Additionally to the hypothesis of Theorem 4, if we suppose that the mapping a satisfies the (U) condition, then the function A has exactly one fixed point.

We skip the proof since it is verbatim of the proof Theorem 3.

\section{Conclusions}

As we derive Theorem 4, by letting some $\beta_{i}=0$, for distinct combinations of $i \in\{1,2,3,4\}$ in Theorem 2, we get some more corollaries of Theorem 2, and also consequences of Theorem 3. Thus, 
we deduce that the main results of the paper cover several existing results in the literature, e.g., [1,5,9]. In particular, by letting $\alpha(p, q)=1$ and $r=1$ in Theorem 5, we obtain the analogue of the renowned result of Boyd-Wong [9] in context of quasi-metric spaces. Notice also that by letting $\beta_{1}(t)=k t, k \in[0,1)$, we get a variant of Banach contraction principle in the setting of quasi-metric spaces.

Author Contributions: Writing-original draft preparation A.F.; writing-review and editing, A.F. and E.K. All authors have read and agreed to the published version of the manuscript.

Funding: This research received no external funding.

Acknowledgments: The authors thank anonymous referees for their remarkable comments, suggestions, and ideas that help to improve this paper.

Conflicts of Interest: The authors declare no conflict of interest.

\section{References}

1. Wong, C.S. Generalized contractions and fixed point theorems. Proc. Am. Math. Soc. 1974, 42, 409-417. [CrossRef]

2. Dung, N.V. Remarks on quasi-metric spaces. Miskolc Math. Notes 2014, 15, 401-422. [CrossRef]

3. Karapinar, E.; Romaguera, S. On the weak form of Ekeland's Variational Principle in quasi-metric spaces. Topol. Appl. 2015, 184, 54-60. [CrossRef]

4. Karapinar, E.; Romaguera, S.; Tirado, P. Contractive multivalued maps in terms of Q-functions on complete quasimetric spaces. Fixed Point Theory Appl. 2014, 2014, 53. [CrossRef]

5. Karapinar, E.; Samet, B. Generalized $\alpha-\psi$-contractive type mappings and related fixed point theorems with applications. Abstr. Appl. Anal. 2012, 2012, 793486. [CrossRef]

6. Samet, B.; Vetro, C.; Vetro, P. Fixed point theorems for $\alpha-\psi$-contractive type mappings. Nonlinear Anal. 2012, 75, 2154-2165. [CrossRef]

7. Karapinar, E.; Roldan-Lopez-de-Hierro, A.-F.; Samet, B. Matkowski theorems in the context of quasi-metric spaces and consequences on G-metric spaces. Analele Stiintifice Ale Univ. Ovidius-Constanta-Ser. Mat. 2016, 24, 309-333. [CrossRef]

8. Alqahtani, B.; Fulga, A.; Karapinar, E. Fixed Point Results On $\Delta$-Symmetric Quasi-Metric Space Via Simulation Function With An Application To Ulam Stability. Mathematics 2018, 6, 208. [CrossRef]

9. Boyd, D.W.; Wong, J.S.W. On nonlinear contractions. Proc. Am. Math. Soc. 1969, 20, 458-464. [CrossRef]

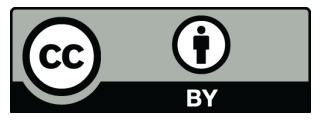

(C) 2020 by the authors. Licensee MDPI, Basel, Switzerland. This article is an open access article distributed under the terms and conditions of the Creative Commons Attribution (CC BY) license (http:/ / creativecommons.org/licenses/by/4.0/). 